\title{
Dental plaques: microbial community of the oral cavity
}

\begin{abstract}
Dental plaques are the biofilms or the microbial population associated with the oral cavity and is responsible for several clinical conditions that affect the host system. Despite the fact that, the oral micro flora offer certain benefit to the host by offering protection to the epithelial cell from damage and enhances the digestion of some substrates, their harmful consequences cannot be denied. The oral cavity dwells over 700 different species of bacteria and is described as one of the most intricate ecosystem. Though majority of them are considered as commensally, some of them are responsible for oral infections ranging from cavities to periodontal diseases and gum related infections. The current review attempts to provide a better understanding of these bacterial communities of the oral cavity. The review provides the insights on dental plaque in general followed by the formation of these dental plaques and the stages involved in the growth and maturation of these dental biofilms. A wide range of micro flora is associated with dental plaques and the review has Attempted to list out the bacterial community at the respective clinical condition and their classification based on their location and stage of infection. The components and the consequences of the dental biofilms have addressed. The review also attempts to disclose the significance of molecular biological methods in improving the identification and characterization of the oral microbial community and has also focused on the possible treatment for dental plaques.
\end{abstract}

Volume 4 Issue I - 2017

\author{
Ranganathan Vasudevan \\ School of Chemical and Biotechnology, SASTRA University, India
}

Correspondence: Ranganathan Vasudevan, School of Chemical and Biotechnology, SASTRA University, Thanjavur- 613 40I, India,Tel 9|-8I21 I|9692,Email vasudevan123@gmail.com

Received: September 02, 2016 | Published: January 31, 2017

Keywords: dental plaques, dental biofilms, oral micro biota, gram positive and negative bacteria, dental plaque diseases

\section{Introduction}

The scientific investigators and researchers over the last few decades have attempted to disclose the significance of the microbial populations and the intricate activities associated with these communities. It is a very interesting fact about these microbes that they have the ability to engineer their system according to the ecosystem they are confined to as a consequence of a series of internal metabolism which includes the contribution from their genetic makeup. This is one of the reasons that make these minute creatures exigent when it comes to the aspect of wiping them out through a suitable remedy because the earlier method will be outdated for the second time due to the extent of adaptability exhibited by the microbe. This aspect of these minute creatures makes them a study of research as it reveals possible measures of safe guarding the human society from the threats posed by the microbial community. ${ }^{1}$ The current review would attempt to focus on one such aspect which involves the bacterial community of the oral cavity and its significance. Dental plaque is a common term associated with the oral microbial community which comprises of the biofilms or a population of bacteria that invade the mouth and can result in a series of health concerning issues. ${ }^{2}$ These plaques can be found within the surfaces of the mouth and are described as sticky color less deposit in the beginning but later on transforms in to a tartar sometimes referred to as calculus which is brown to pale yellow in appearance and can be found between the teeth, on the front and back of the teeth and all along the gum line. Researchers have regarded dental plaque as microbial plaque, oral/dental biofilms as well as bacterial plaque biofilms. ${ }^{2}$ Though the plaque is usually associated with the oral diseases which include cavities and other gum diseases, its construction is normal which cannot be prevented. Dental plaques can result in tooth decay which as a consequences leads to tissue damage due to the acidic secretion of the bacteria. These dental biofilms can be quite serious as their acidic nature causes the demineralization of the teeth resulting in the formation of calculus which is also known as tartar. These are hardened structures that require immediate attention through professional cleaning as they cannot be removed by tooth brush. It is therefore very essential to eradicate these dental plaques to avoid the formation of dental caries and related gum disease. ${ }^{3,4}$ Research studies have confirmed the structural and functional organization of these dental biofilms and are diverse in terms of their microbial composition. Though the dominant type found in the infected sites are diverse from those found in uninfected sites, the alleged perpetrators can be detected in low numbers at regular sites. ${ }^{5}$ Studies have disclosed the role of mutant species of streptococcus and lactobacilli in dental caries due to their ability to tolerate acidic environment. However, the involvement of other species with relevant traits cannot be denied. One of the most effective strategies of controlling the dental plaques is by eliminating biofilm formation.

\section{Formation of dental biofilms}

Biofilms are microbial communities that grow on living and non living surfaces which range from a wooden plank or any other material to human tissues. They are highly complex and organized structures that initially exist as a plank tonic cell. After attachment they pass through a series of developmental stages which result in growth and multiplication leading to the formation of mature cells capable of causing the infection. These biofilms also play a significant role in making these enclosed bacteria resilient to a range of antimicrobial agents by offering them protecting. ${ }^{6,7}$ One of the most widely studied areas of bacterial biofilms includes the dental plaques otherwise referred to as oral biofilms or bacterial plaque biofilms which has been a scientific challenge to the medical and pharmaceutical industry. The bacterial community within the oral cavity is considered to be very complex and highly diverse comprising of over 700 different species. The environment within the mouth is vital as warmth; moisture and neutral $\mathrm{pH}$ favor the growth of microorganisms. ${ }^{8}$ The microbial composition of the normal plaque biofilms significantly differs from this bacterial community confined to carious regions and in the sub- 
gingival regions. The reason for this diversity is due to the presence of different set of genes when compared to free floating bacteria. As a matter of fact, a biofilm producing bacterial community is different as it facilitates major changes in bacterial behavior. These behavioral changes could include their interaction with the other microbes and the host organism in addition to their response to the environmental conditions. ${ }^{9}$

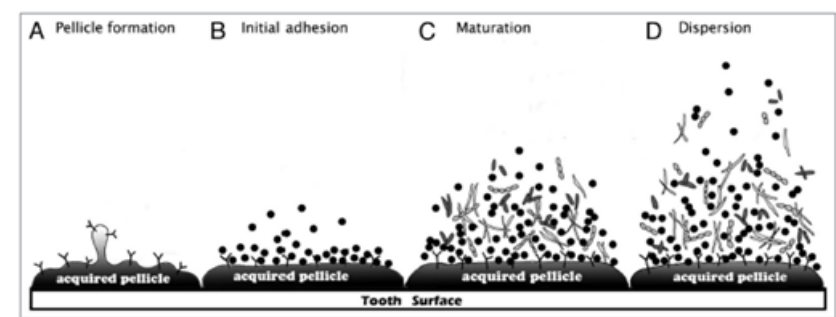

Figure I Depicts the formation of dental biofilms on tooth surface. ${ }^{8}$

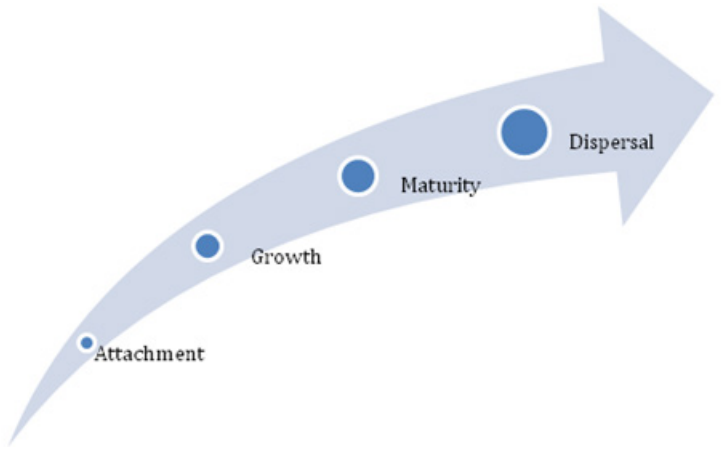

Figure 2 Schematic representation of different stages of bacterial plaque biofilm formation.

Stages of dental biofilms formation.

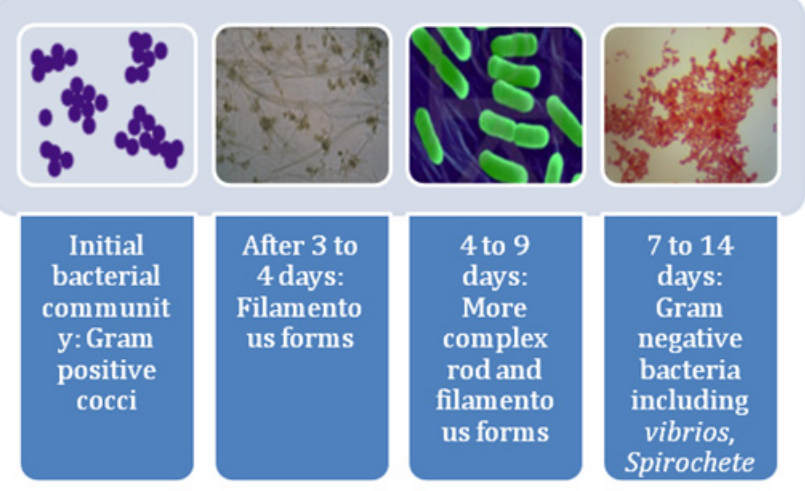

Figure 3 Diagrammatic representation of bacterial occurrences at various phases of plaque maturation. ${ }^{20,21}$
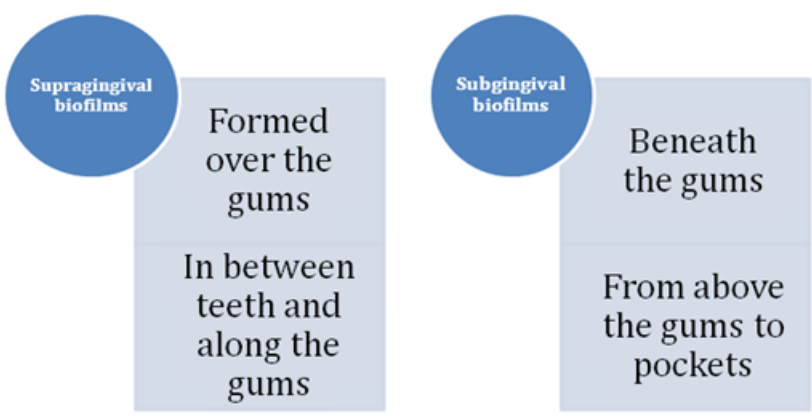

Figure 4 Plaque classification based on location in the oral cavity 22,23 Dental biofilms

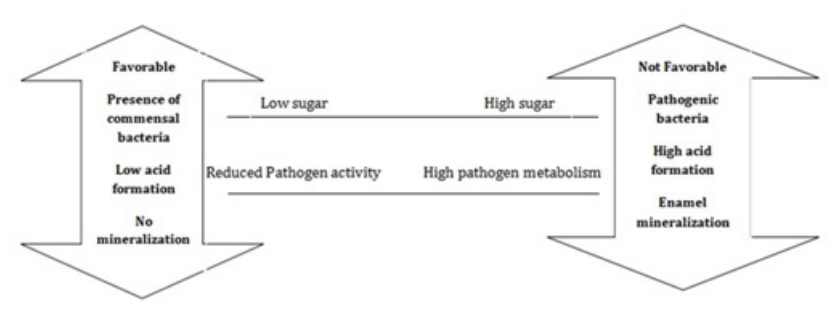

Figure 5 Depicts the environmental shifts resulting in the accumulation of pathogenic bacteria. ${ }^{46}$

The above figure outlines the various stages involved in the formation of bacterial biofilms associated with the oral cavity. The figure illustrates the various stages that range from pellicle formation followed by initial adhesion, maturation and finally resulting in the dispersal of the mature biofilms. The ability of these plaques to resist the antimicrobial agents is an interesting feature and this has confirmed the use of mechanical means for plaque removal rather than conventional methods.

Formation of dental biofilms is an intricate phenomenon involving various stages which begins with adsorption on to the tooth surface succeeded by passive transfer of bacteria initiated by weak long range force of attraction which is subsequently favored by covalent and hydrogen bonds which make the attachment irreversible. The extent of biofilm formation also relies on the degree of similarity between the bacterial communities. The attraction between the same species occurs due to auto aggregation whereas co-aggregation allows the attraction between different species. ${ }^{10}$ The glycoprotein's present in the saliva play a significant role as they bind to the surface of the teeth and this result in the formation of pellicle. The pellicle serves as a binding site for the organism. Most of the Gram positive species like Streptococcus, Actinomyces, Haemophilus, Neisseria and Veillonella are regarded as primary colonizers of the pellicle. ${ }^{11}$ This phase is known as adhesion phase or the attachment phase where the microbe adheres to the surface of the teeth. This phase is also known as lag phase because of reduced metabolic activity but it is not inert. After the initial attachment, secretion of extracellular matrix grips the bacterial communities together which is followed by the invasion of secondary colonizers. Co-aggregation favor this process resulting in the functional organization of dental biofilms and it leads to the formation of various morphological structures refereed as corncobs and rosettes. The bacterial population within the extracellular matrix multiplies resulting in a diverse mixed biofilms. ${ }^{10}$ Several studies have also illustrated the importance of the products produced by the primary colonizers in favoring the secondary colonizers and studies have also demonstrated the shift of biofilm composition from Gram positive bacteria to Gram negative bacteria. Scientific experiments have validated the significance of $\mathrm{N}$-acetylmuramic acid produced by Gram positive bacteria which favors Tanerella (Gram negative bacteria). In addition to Tanerella, the other Gram negative bacterium includes orphyromonas gingavalis and Fusobacterium nucleatum. The secondary colonizers have indeed enhanced the diversity of the dental biofilms. ${ }^{6}, 12$ After the initial attachment (lag phase) and the exponential growth phase (log phase), the cells are ready to be detached after maturity.

It is quite obvious from the above sections that the bacterial are the prime perpetrators and the initial adherence is facilitated by Gram positive bacteria and later on enhances the attachment of Gram negative bacteria resulting in a complex mixed population. The bacterial communities associated with the oral cavity are enclosed within the extracellular matrix that keeps these organisms protected 
against different external factors including antimicrobial agents. ${ }^{14}$ This ability of the bacteria differentiates it from the other free floating non biofilms forming species. Research investigations have also highlighted the significance of biofilms in enhances the behavioral changes among the organisms which in turn facilitate vital processes like cell to cell communication as well as the organism's interaction with the host. These altered activities as a consequence of biofilm formation has enhanced the scope of understanding the impact of these dental plaques on the overall health of the individual rather than just the oral cavity. ${ }^{15}$

\section{Components of dental plaques}

The oral cavity comprises of a variety of bacteria in addition to leukocytes, neutrophils, macrophages and lymphocytes which contribute positively towards the individual's health. It is estimated that the major portion of the plaques are made up of water of which bacteria accounts to about $70 \%$ (that slightly less than 3/4th) and polysaccharides and glycoproteins forms the remaining 30\%. Several research studies have validated the role of Streptococcus mutants and some anaerobic bacteria in the initial attachment on the tooth surface and hence are regarded as primary colonizers and they are responsible for the formation of premature biofilm community. ${ }^{16,17}$

Table I The table below represents the various Gram positive and negative bacteria associated with the dental plaques. ${ }^{13}$

\begin{tabular}{ll}
\hline Clinical Observable Fact & Key Organism \\
\hline & Primary colonizers (mostly Gram positive \\
bacteria) & Streptococcus \\
& Actinomyces \\
& Haemophilus \\
& Neisseria \\
Veillonella & Secondary colonizers (mostly Gram negative \\
Plaque Formation & bacteria) \\
& Porphyromonas \\
& Fusobacterium \\
Streptococcus mutants \\
Streptococcus sanguinis \\
Lactobacilli \\
Porphyromonas \\
Actinobacillus \\
Preponema \\
Selenomonas \\
Tannerella \\
\end{tabular}

\section{Bacteria}

Streptococcus mutants and some anaerobes like Fusobacterium and actinobacteria initially adhere to the tooth surface and their composition significantly varies based on the location in the mouth The bacteria associated with the dental plaques are those that naturally occur in the oral cavity but the reason for their accumulation in the oral cavity is due to the failure of removing the plaques through regular brushing. These bacteria on the tooth surface are capable of fermenting the dietary sucrose resulting in the production of acids which in turn worsens the condition.

The above classification depicts the subclasses of biofilms based on the location of their occurrence. The biofilms are classified as supra gingival and sub gingival on the basis of locality in the oral cavity. In the former case the plaques are formed on the gums which occur after brushing and this is considered as the first plaques that can be found in between the teeth, in the grooves and by the side of the gums. These usually comprise of aerobic bacteria which means that oxygen is a vital factor for these bacteria to survive. As the time progresses the anaerobic bacteria invades the plaque. In contrast, the sub gingival plaques are found beneath the gums as a consequence of downward growth of the bacteria and comprises of anaerobic bacteria. ${ }^{19}$

\section{Oral environment}

The growth and maturity of the dental plaque is due to tepid and wet environment of the oral cavity. This in turn favors the exponential rise in the bacterial load. Vital aspects that decide the fate of the dental plaques are $\mathrm{pH}$, saliva, temperature and certain chemical reactions of the oral cavity like the oxidation-reduction reaction. ${ }^{22,23}$ The $\mathrm{pH}$ range of 6 to 7 in saliva is considered to be normal and any deviation from this range favors the plaque biofilm formation. This provides evidence which confirms that the innate surroundings of the oral cavity serve as an ideal platform for the growth and development of the bacteria. ${ }^{24,25}$ Several studies have illustrated the significance of the saliva as a buffer in maintaining the $\mathrm{pH}$. On the other hand, nutrients including proteins and amino acids in saliva nourish the bacteria in plaque formation. In addition to $\mathrm{pH}$, another vital factor is the temperature as a slightest change can result in the relocation of the dominant species. The normal temperature of the oral cavity ranges from 35 to $360 \mathrm{C}$. Chemical reactions of the oral cavity also favor the plaque biofilm formation. The redox reactions that are carried out by the aerobic bacteria maintain the oxygen at a stable level which in turn favors the bacterial biofilms to survive. ${ }^{23}$

\section{Consequences of dental plaques}

It is quite evident that the oral environment provides the vital nutrients that favors the growth and maturity of the bacterial community and their association certainly leads to issues of health concern. The infection of the gingival tissue is known as gingivitis where the bacteria is responsible for the inflammation of the tissue. The symptoms include redness, swollen appreance and bleeding of gums. This dental contagion can be reversable in the initial stages but if left untreated it progrsses in to a stage known as periodontal disease otherwise referred as periodontitis. ${ }^{26,27}$ This is the infection of the gums and causes the bone damage. The organsisms associated with this infection are known to produce certain enzymes that are capable of damaging the bone. ${ }^{28}$ Acids produced from the dental plaque causes the demineralization of the adjecent tooth resulting in dental caries. Scietific investigators have disclosed several health issues as a consequence of dental plaques and have in fact considered dental decay as one of the most widespread contagion globally. Certain studies have highlighted the importance of a variety of factors like the genetic makeup of the organism, immunological aspects and the its behavioral aspects with the host environment. ${ }^{29}$

Though the infection is common in all age groups, children are highly vulnarable as several studies have illustrated a high level of dental caries in younger children. In fact, this condition is considered to be an issue of health concern among children globally which accounts to about $50 \%$ of children withih the age gropu of 9 and the rate of occurrence increases as the age progresses. As a matter of fact, dental care is one of the most unsatisfied need that's yet to be achieved. ${ }^{30}$ The microbiological approaches to counter act the dental infections are narrow due to the lack media that is very selective as well as the nature of the organisms that are difficcult to cultivate. Despie the fact, that many research studies have accused Streptococcus mutan and Latobacillus as prime perpatrators, use of culture free procedures have disclosed the aassociation of over 700 species. Scientific surveys 
of the microbial variety of the human oral cavity based on culture free clonal analysis of gene sequences have shown a higher degree of microbial load associated with the oral cavity. ${ }^{31}$

Many studies have provided scientific evidences that claim the prevalence of oral infection as a consequence of altered host homeostasis and this in turn leads to over growth of the pathogen or reduces the load of opportunistic pathogens. Advancements in the area of molecular biology and molecular techniques have enhanced the understanding of the bacterial community associated with the oral cavity and their means of communication. ${ }^{32-34}$

\section{Significance of molecular approaches in understanding oral micro biota}

As mentioned in the earlier section, the advancement in the area of molecular biology has certainly contributed positively in understanding the oral microbial load. The human body dwells a variety of microorganisms that collectively forms the micro flora invading the gut, vagina and the oral cavity. The microbial community associated with the different parts of the human body has been a topic of scientific interest and scientists have been attempting to disclose various hidden facts of scientific significance. One reason for this is due to the extent of threat caused as a consequence of these microbial invasions on the host health and the medical community as well. Available evidences from different surveys and demonstrative studies point towards the metabolic functions of the organism and its impact on the host system. ${ }^{35,36}$ For instance, the significance gut microbial load in digesting substrates and protecting epithelial cell against injury is the affirmative aspect of the organism but the advancement in scientific procedures has enabled to comprehend their possible role in diseases. ${ }^{37}$ Molecular biology techniques have served as the best means to categorize the different organisms within the community associated with the host including the oral microbial community. Before the expansion of molecular techniques, such tasks of identifying and categorizing the bacterial community relied on the ability to culture and classify the organism. ${ }^{33}$ Several demonstrative studies have validated the existence of physical and metabolic interaction between the microorganisms within the same community. This in turn enables the persistence of certain organisms as the earlier sections have discussed the significance of products of primary colonizers providing the necessary ingredients for the survival of the secondary colonizers. ${ }^{38}$ Studies have also confirmed that the microbes benefitted through these complex interactions are recalcitrant and are not easy to cultivate but biological approaches have simplified this task through gene sequencing methods. The culture independent 16S rRNA sequencing techniques has enhanced the understanding of human oral microbial load and enabled the cultivation of recalcitrant species. ${ }^{33}$ These gene based approaches have disclosed the absolute degree of microbial diversity associated with the oral cavity. The microbial load of the human mouth is considered to be highly complex comprising of over 700 bacterial species and the diversity of the invading microorganism includes Achaea, protozoa, and fungi in addition to bacteria. ${ }^{39,31}$ Presence of Achaea is regarded as an appealing trait by many scientific investigators and has enhanced the oral microbial diversity.

These organisms are considered as the primitive prokaryotes found at the ocean floor and their association with the oral micro flora has fascinated the research community but their role in the microbial community is yet to be determined. This degree of microbial diversity has improved to comprehend the role of genetic information in compromising the host's health and disclose the significance of the bacterial genetics in making them tough. As a matter of fact, the oral micro flora comprising of a variety of bacterial species is a pool of millions of genes because an average bacterial species consists of 2000-6000 genes. When this amount of genes can be present in a single bacterial species, it is quite obvious that this number would reach several folds in a bacterial community comprising of hundreds of species. This genetic information reveals several hidden facts including metabolic pathways, microbial secretions that have the ability to regulate the surroundings and the factors that might compromise the host defense system. ${ }^{40}$

\section{Possible treatment}

The micro flora dwelling the oral cavity are usually natural, highly organized and interactive. The bacterial community associated with the mucous and dental surface comprises of multi species biofilms and are commensally in nature. They are known to play a vital role in the normal functioning of the host physiology and avoid the colonizing of unwanted microorganisms. The existence of a self-motivated balance between the micro flora and the host is very significant as the organisms contribute positively towards the host system. But when there breach in this balance the host system is compromised as a consequence of increase in microbial load resulting in the outbreak of diseases. ${ }^{41}$ Hence there is a need for proficient plaque control techniques and effective treatments to sustain the normal dental micro biota at the desired levels that is well-suited in order to retain the favorable assets of these bacterial communities. The prime objective is to retain the desirable levels of the microbial load that is beneficial to the host organism and to condense the dental risks as a consequence of plaque accumulation. Antimicrobials and anti plaque agents enhances the plaque regulation using direct and indirect methods which not only eliminates the infective dental biofilms but also prevents the bacterial growth and metabolism at lower concentrations. ${ }^{42}$ The antibacterial and anti plaque agents have been useful and demonstrative studies have substantiated that the treatment is possible for targeting the organism as well as the factors that serve as driving force in the growth and development of the dental biofilms. There are certain facts that have to be held in reserve prior to the treatment as these facts have been validated by several researchers.

i. Natural existence of oral micro flora and it benefits the host organism.

ii. Disease is a consequence of a breach in the balance between the microbial load and the host organism.

iii. Local environment can lead to this breach.

iv. Disease can be averted by eliminating the undesirable biofilms and sustaining the required level of microbial load in the oral cavity.

v. Microbial homeostasis maintenance promotes oral health.

vi. Efficient plaque control measures of oral cavity is not easy and may not be enough for the number of patients but researchers have hypothesized the following strategies as possible means of treating the disease.

vii. Reduction in acid production.

viii. Elimination of proteolytic and anaerobic bacteria.

\section{ix. Encourage beneficial bacteria.}

Acid production from the microbial metabolism of dietary sugars and low $\mathrm{pH}$ in dental biofilms causes several dire consequences. Lower $\mathrm{pH}$ favors the growth of acid producers that are acid tolerant and causes the demineralization of teeth tissues and also inhibits 
the growth of commensally bacteria that are beneficial to the host The prolonged condition of lower $\mathrm{pH}$ increases the load of acid producers which disrupts the natural balance between the oral micro flora and the host and enhances the risk of caries. Comparable effects were demonstrated when the composition of saliva was altered as a consequence of medical side effects. ${ }^{44,45}$ Suitable approach can be used to reduce the acid production in order to counter act the harmful effects. Fluoride has been proven to be beneficial in restoring the condition as it promoted the remineralization and prevents demineralization. Studies have also confirmed the role of fluorides in inhibiting the sugar metabolism, sugar transport and glycolysis. On the other hand, antimicrobials employed at sub lethal levels are known to inhibit the process of sugar metabolism and reduces the acid production. ${ }^{46}$

Sugar substitutes instead of dietary sugar are a better option as these cannot be directly broken down by bacteria and this in turn reduces the acid production. Supplements like arginine or urea can be used that are metabolized by plaque bacteria to alkali which in turn increases the $\mathrm{pH}$ which inhibits the acid producers ultimately reducing the acid production. ${ }^{47,48}$ In silico approaches have been used to validate the significance of $\mathrm{pH}$ based on the degree of acid buffering capacity. In addition, antimicrobial agents at nominal concentration have been beneficial in eliminating the undesirable biofilms and can also be selective by sustaining the natural balance in the oral cavity.

Most of the bacteria associated with the dental plaques are anaerobic and are capable of braking down proteins. These anaerobic and proteolytic bacteria require a constant supply of vital factors like the proteins and other cofactors. Such pathogens can be eliminated by altering the environment as it creates an unfavorable condition for the pathogen to survive. Several approaches like the use of redox agent, anti inflammatory agents and antimicrobial agents have been investigated. Redox agents increase the $\mathrm{pH}$ within the oral cavity and prevent the infection of the periodontal pocket. ${ }^{49}$ Resolvins and lipoxins are the novel anti inflammatory agents that have the ability to heal the damaged tissue and also reduce the gingival cervicular fluid flow (GCF) which in turn keeps the flow of essential nutrients out of the bacteria's reach and inhibits the growth and development. ${ }^{50,51}$ Antimicrobial agents have also been useful in preventing the plaques at marginal concentration. Triclosan which is a widely prescribed mouthwash acts as an antimicrobial and anti metabolic agent due to its ability of inhibiting microbial sugar metabolism and protease activity. In addition, it also acts as an anti inflammatory agent and enhances the healing of the damaged tissues. ${ }^{52,53}$

The methods mentioned in the earlier section not only eliminate the unwanted plaque biofilms but also restores the condition by maintaining the natural ecological niche in the oral cavity. Apart to the above strategies, additional approaches such as use of prebiotics have been beneficial and several studies have highlighted the significance of prebiotics as they are very selective and promote the growth of beneficial organism. Prebiotics encouraging the growth of certain oral bacteria that are beneficial to the oral cavity can be employed. The beneficial effects would be the development of functional foods that contribute affirmatively towards the oral health besides nutritional fulfillment. ${ }^{54}$ Molecular biology has its own significance and this field can be used to create non pathogenic strains through molecular techniques. Identification of a suitable oral prebiotics strain or designing a non virulent strain can be used to prevent the colonization of harmful or pathogenic strains. A strategy known as the replacement therapy can be used which uses molecular techniques to engineer a strain that can be beneficial. For instance, scientific studies have been carried out which has demonstrated the production of a wild type strain of Streptococcus mutants that cannot produce lactic acid and are capable of producing bateriocin that restrains the growth of natural Streptococcus mutants. ${ }^{55}$ Researchers have also attempted to disclose the significance of probiotics in oral application but this is debatable and yet to be validated. Probiotics in connection with the oral application is considered to be controversial due to type of strains involved. Most of the evaluated strains belong to lactobacilli or Bifidobacteria which have been associated with dental caries. However, the truth about their ability to colonize the oral cavity is again debatable and yet to be disclosed. Re-implantation of local bacteria that is a resident of the oral cavity in to the gum pockets has been proved useful and enhances tissue healing and resistance. ${ }^{56,48}$

\section{Conclusion}

It well understood from the past research demonstrations that dental plaques are biofilms of oral cavity comprising of over 700 different species of bacteria and are responsible for a series of health issues of the oral cavity ranging from the infection of the teeth to the infection of the gums finally resulting in chronic clinical conditions. Both Gram positive and negative bacteria are embedded in the plaque biofilms and the environmental conditions are vital for the survival of these bacterial communities. Though the micro biota of the oral cavity are natural and considered to be commensally, their harmful effects cannot be denied. The disruption of the natural balance between the organisms and the host organism causes the accumulation of pathogenic bacteria resulting in damage of the teeth and gum tissues. Advancements in molecular techniques has indeed enhanced the level of identification and characterization of oral micro biota as these organisms are usually considered to be recalcitrant and are uncultivable. However, independent gene sequencing techniques has narrowed this task and has improved the understanding of the plaque biofilms. Though several studies have disclosed different means of measures to counter act the plaque biofilms, further studies to validate the facts are required.

\section{Acknowledgments}

None.

\section{Conflicts of interest}

None.

\section{Funding}

None.

\section{References}

1. Marsh PD. Dental Biofilms in Health and Diseases. Springer International Publishing, USA. 2016. p. 41-52.

2. Darby ML, Walsh MM. Dental Hygiene Theory and Practice. (4th edn). 2010. p.1192.

3. Wolf H, Hassell T. Color Atlas of Dental Hygien. Thieme New York, 333 Seventh Avenue, New York, USA. 2006. p.351.

4. Verkaik M, Busscher H, Jager D, et al. "Efficacy of natural antimicrobials in toothpaste formulations against oral biofilms in vitro". J Dent. 2011;39(3):218-224.

5. Marsh PD. Dental plaque as a biofilm and a microbial communityimplications for health and disease. BMC Oral Health. 2006;6(Suppl 1):S14.

6. Gurenlian J R. The Role of Dental Plaque Biofilm in Oral Health Journal of Dental Hygiene. 2007; 81:116-116. 
7. Huang R, Li M, Gregory RL. Bacterial interactions in dental biofilm. Virulence. 2011;2(5):435-444.

8. Marsh PD, Moter A, Devine DA. Dental plaque biofilms: communities, conflict and control. Periodontol. 2011;55(1):16-35.

9. Hao $\mathrm{W}, \mathrm{Xu} \mathrm{H}$, Chen $\mathrm{X}$, et al. Changes in Dental Plaque Microbial Richness and Oral Behavioral Habits during Caries Development in Young Chinese Children. Caries Res. 2015;49(2):116-123.

10. Quirynen M, Teughels W, Haake SK, et al. Microbiology of Periodontal Diseases. In: Newman MG, Takei HH, et al. (Eds.), Carranza's Clinical Periodontology. (10th edn), Elsevier (Saunders), St Louis, Missouri, USA. 2006. p.134-169.

11. Vir K. Oral biofilms and host immune response. 2010.

12. Spratt DA, Pratten J. Biofilms and the Oral Cavity. Re/Views in Environmental Science and Bio/Technology (2003). 2003;2:109.

13. https://microbewiki.kenyon.edu/index.php/File:Wiki_table.png

14. Sbordone L, Bortolaia C. "Oral microbial biofilms and plaque-related diseases: microbial communities and their role in the shift from oral health to disease". Clin Oral Investig. 2003; 7(4):181-188.

15. https://microbewiki.kenyon.edu/index.php/Dental_Plaque_Biofilms

16. Marsh PD, Bradshaw D J. "Dental plaque as a biofilm". J Ind Microbiol. 1995;15(3):169-175.

17. Kolenbrander PE. "Oral Microbial Communities: Biofilms, Interactions, and Genetic Systems”. Annu Rev Microbiol. 2000;54(1):413-437.

18. https://en.wikipedia.org/wiki/Dental_plaquecite_noteDarby_M_L_2010-1

19. Wilkins E. Clinical Practice of the Dental Hygienist. Wolters Kluwer Health/Lippincott Williams \& Wilkins, (10th edn), Philadelphia, USA. 2009.

20. Chetrus V, Ion I R. "Dental Plaque - Classification, Formation, and Identification." Int J Med Detistry. 2013;3(2):139-143.

21. Garcia Godoy F, Hicks MJ. "Maintaining the Integrity of the Ename Surface: The role of dental biofilm, saliva, and preventative agents in the enamel demineralization and remineralization". J Am Dent Assoc. 2008;139(suppl 2):25S-34S.

22. Marsh PD. "Are dental diseases examples of ecological catastrophes?" Microbiology. 2003; 149(2):279-294.

23. Marsh PD, Devine DA. «How is the development of dental biofilms influenced by the host?» J Clin Periodontol. 2011;38 (s11):28-35.

24. Humphrey SP, Williamson RT. A review of saliva: normal composition, flow, and function. J Prosthet Dent. 2001;85(2):162-169.

25. McDermid AS, McKee AS, Marsh PD. "Effect of Environmental pH on Enzyme Activity and Growth of Bacteroides gingivalis W50". Infect Immun. 1998;56(5):1096-100.

26. Parakrama Chandrasoma, Clive R Taylor. «Part A. General Pathology, Section II. The Host Response to Injury, Chapter 3. The Acute Inflammatory Response, sub-section Cardinal Clinical Signs». Concise Pathology (3rd edn), NY McGraw-Hill, New York, USA. 2005.

27. Noble SL. Clinical Textbook of Dental Hygiene and Therapy. (2nd edn), Wiley-Blackwell, West Sussex. 2012. p.96-97.

28. Tonetti MS, Eickholz P, Loos BG, et al. "Principles in Prevention of Periodontal Diseases". J Clin Periodontol. 2015;42 (Suppl16):S5-11.

29. Peterson SN, Snesrud E, Liu J, et al. The Dental Plaque Microbiome in Health and Disease. PLOS ONE. 2013;8(3):e58487.

30. Rockville. Oral health in America: a report of the Surgeon General Journal of the California Dental Association. 2000;28:685-695.
31. Dewhirst FE, Chen T, Izard J, et al. The human oral microbiome. $J$ Bacteriol. 2010;192(19):5002-5017.

32. Darveau RP. Periodontitis: a polymicrobial disruption of host homeostasis. Nat Rev Microbiol. 2010;8(7):481-490.

33. Kuramitsu HK, He X, Lux R, et al. Interspecies interactions within oral microbial communities. Microbiol Mol Biol Rev. 2007;71(4):653-670.

34. von Fraunhofer JA, Loewy ZG. Factors involved in microbial colonization of oral prostheses. Gen Dent. 2009;57(2):136-143.

35. Human Microbiome Project Consortium. Structure, function and diversity of the healthy human microbiome. Nature. 2012;486(7402):207-214.

36. Turnbaugh PJ, Ley RE, Hamady M, et al. The human micro biome project. Nature. 2007; 449(7164):804-810.

37. Elinav E, Strowig T, Kau AL. NLRP6 inflammasome regulates colonic microbial ecology and risk for colitis. Cell. 2011;145(5):745-757.

38. Xuesong He, Jeffrey S McLean, Anna Edlund, et al. Cultivation of a humanassociated TM7 phylotype reveals a reduced genome and epibiotic parasitic lifestyle. Proc Natl Acad Sci USA. 2015;112(1):244-249.

39. Avila M, Ojcius DM, Yilmaz O. The oral microbiota: living with a permanent guest. DNA Cell Biol. 2009;28(8):405-411.

40. Rajendhran J, Gunasekaran P. Microbial phylogeny and diversity: Small subunit ribosomal RNA sequence analysis and beyond. Microbiol Res. 2011;166(2):99-110.

41. Kistler JO, Booth V, Bradshaw DJ, et al. Bacterial community development in experimental gingivitis. PLOS ONE. 2013;8(8):e71227.

42. Marsh PD. Contemporary perspective on plaque control. $\mathrm{Br}$ Dent $\mathrm{J}$. 2012;212(12):601-606.

43. Head DA, Marsh PD Devine DA. Non-lethal control of the cariogenic potential of an agent-based model for dental plaque. PLOS ONE. 2014;9(8):e105012.

44. Wolff A, Zuk-Paz L, Kaplan I. Major salivary gland output differs between users and non-users of specific medication categories. Gerodontology. 2008;25(4):210-216.

45. Deboni AL, Giordani AJ, Lopes NN, et al. Longterm oral effects in patients treated with radiochemotherapy for head and neck cancer. Support Care Cancer. 2012;20(11):2903-2911.

46. Marsh PD. Controlling the oral biofilm with antimicrobials. J Dent. 2010;38(Suppl.1):S11-15.

47. Gordan VV, Garvan CW, Ottenga ME, et al. Could alkali production be considered an approach for caries control?. Caries Res. 2010;44(6):547-554.

48. Burne RA, Marquis RE. Alkali production by oral bacteria and protection against dental caries. FEMS Microbiol Lett. 2000;193:1-6.

49. Wilson M, Gibson M, Strahan D, et al. A preliminary evaluation of the use of a redox agent in the treatment of chronic periodontitis. J Periodont Res. 1992;27(5):522-527.

50. van Dyke TE. The management of inflammation in periodontal disease. J Periodontol. 2008;79(8 Suppl):1601-1608.

51. Hasturk H, Kantarci A, van Dyke TE. Paradigm shift in the pharmacological management of periodontal diseases. Front Oral Biol. 2012;15:160-176.

52. Barros SP, Wirojchanasak S, Barrow DA, et al. Triclosan inhibition of acute and chronic inflammatory gene pathways. J Clin Periodontol. 2010;37(5):412-418.

53. Wallet MA, Calderon N, Alonso TR, et al. Triclosan alters antimicrobial and inflammatory responses of epithelial cells. Oral Dis. 2013;19(3):296-302. 
54. Devine DA, Marsh PD. Prospects for the development of probiotics and prebiotics for oral applications. J Oral Microbiol. 2009;1:3402.

55. Hillman JD. Replacement therapy for dental caries. In: Newman HN Wilson M (Eds.), Dental plaque revisited: oral biofilms in health and disease. Cambridge, USA. 1999. p.587-599.
56. Teughels W, Newman MG, Coucke W, et al. Guiding periodontal pocket recolonization: a proof of concept. J Dent Res. 2007;86(11):1078-1082. 